\title{
Research Paper: The Investigation of Factors Affecting the Gait of the Pa- tients Suffering From Multiple Sclerosis
}

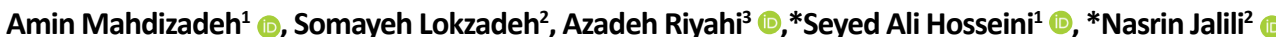

1. Department of Occupational Therapy, University of Social Welfare and Rehabilitation Sciences, Tehran, Iran

2. Department of Occupational Therapy, School of Rehabilitation Sciences, Isfahan University of Medical Sciences, Isfahan, Iran.

3. Department of Occupational Therapy, School of Rehabilitation, Arak University of Medical Sciences, Arak, Iran.

\begin{tabular}{c|l}
$\begin{array}{c}\text { Use your device to scan } \\
\text { and read the article online }\end{array}$ \\
tients Suffering From Multiple Sclerosis (Persian)]. Archives of Rehabilitation. 2019; 20(1):64-73. http://dx.doi.org/10.32598/rj.20.1.64 \\
doittp://dx.doi.org/10.32598/rj.20.1.64
\end{tabular}

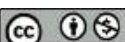

Received: 10 Sep 2018

Accepted: $25 \operatorname{Jan} 2019$

Available Online: 01 Apr 2019
Keywords:

Gait, Muscle strength of lower extremities, Attention, Cognition, Multiple Sclerosis

\section{ABSTRACT}

Objective Multiple Sclerosis (MS) is a chronic inflammatory, demyelinating, and neurodegenerative disorder of the Central Nervous System (CNS) and it is also the most common cause of non-traumatic neurological disability in young adults. MS in women, especially young women, is about 1.5 to 3 times more common than men all over the world. About 2.5 million people worldwide are suffering from MS. The destruction of myelin in certain areas exhibits certain signs and symptoms. Balance disorders are particularly problematic because they are associated with difficulty in moving from one position to another, sustaining an upright posture, and performing functional activities such as walking and turning, all of which predisposes people with MS to the loss of equilibrium and falls. Isfahan City in Iran suffers from a high prevalence of MS that put it amongst the regions with the highest prevalence of MS in Asia and Oceania. This study aimed to use the holistic approaches of occupational therapy to investigate the physical and cognitive factors (attention, cognition and muscle strength of lower extremities) that affect the gait of MS patients in the city of Isfahan, Iran.

Materials \& Methods The present study was a descriptive-correlational one and convenience sampling method was used to recruit the samples. The study population consisted of all patients with MS in Isfahan City in August 2017. A group of 70 patients ( 24 males and 46 females with the Mean $\pm S D$ age of $32.20 \pm 7.55$ y) met the inclusion-exclusion criteria and were enrolled in the study. To be eligible for the study, the patients had to meet the following inclusion criteria: having a neurologist-confirmed diagnosis of MS with MRI scan, being able to read and write, and walk at least $100 \mathrm{~m}$ independently. We excluded patients with cognitive impairments that might hinder understanding of the tasks to be accomplished, those with any comorbid neurologic condition other than MS, and those who are unwilling to collaborate with the research process. After providing sufficient information about the research process and getting informed consent of the patients, the questionnaires and tests were provided to the participants to be completed. The study tools were the Mini-Mental Status Examination (MMSE), $10 \mathrm{~m}$ Walking Test (10 MWT), Timed Up and Go test (TUG), Sit to Stand test, and Stroop test. For data analysis, the Pearson correlation coefficient and multiple regression analysis were performed in SPSS version 16.

Results In this study, 70 patients suffering from MS participated. The results showed a significant negative correlation between the scores of walking speed test (10 $\mathrm{m}$ walking) and the scores of the functional strength of the lower extremities muscles $(P<0.001)$ and attention $(P=0.01)$. There is also a significant correlation between muscle strength of lower extremity and balance $(P=0.01)$. Regression analysis shows that the muscle strength of lower extremities has the highest contributions in predicting the speed of walking in patients with MS.

Conclusion According to the study findings, among the physical and cognitive factors affecting gait and balance such as muscle strength of lower extremity or cognition and attention, the muscle strength of lower extremity is the most important factor affecting the balance and speed of walking in patients with MS that still can walk. In the process of rehabilitation, especially in occupational therapy, special attention must be paid to a muscle strengthening program for the lower extremities of patients suffering from MS.

\section{* Corresponding Author:}

Seyed Ali Hosseini, PhD.

Address: Department of Occupational Therapy, University of Social Welfare and Rehabilitation Sciences, Tehran, Iran.

Tel: +98 (21) 22180037

E-Mail: alihosse@gmail.com

\section{Nasrin Jalili, MSc}

Address: Department of Occupational Therapy, School of Rehabilitation Sciences, Isfahan University of Medical Sciences, Isfahan, Iran. Tel: +98 (311) 7922021

E-Mail: nasrin_jalili@ymail.com 


\title{
بررسى عوامل مؤثر بر راهرفتن افراد مبتلابه بيمارى مولتيبل اسكلروزيس
}

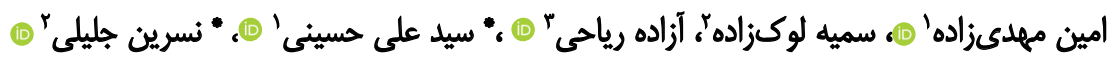

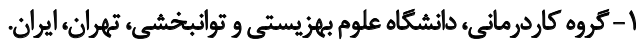

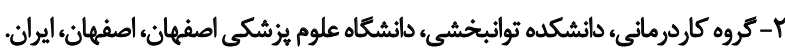

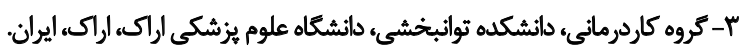

\begin{abstract}
حكS

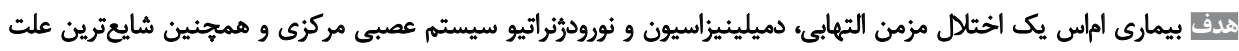

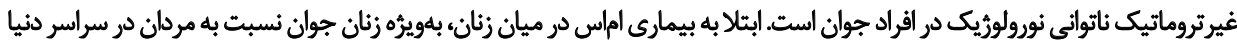

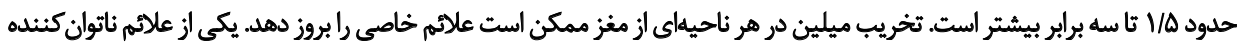

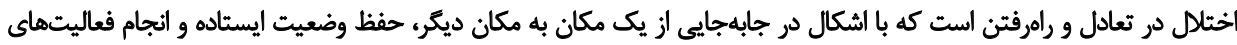

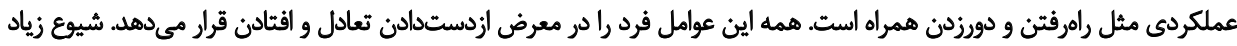

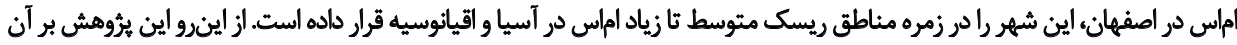

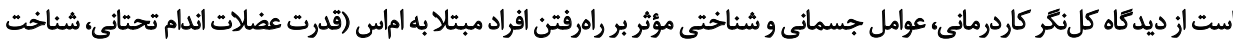

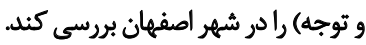

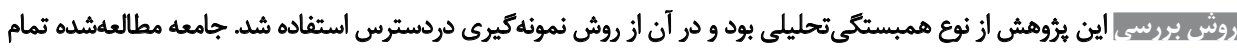

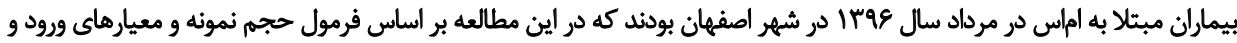

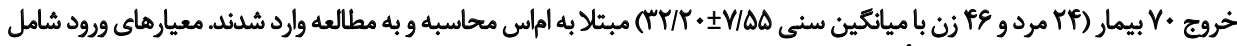

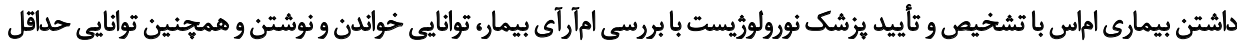

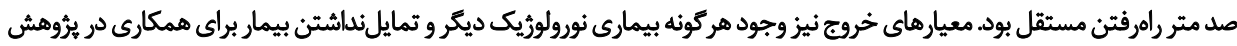

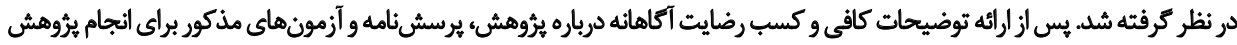

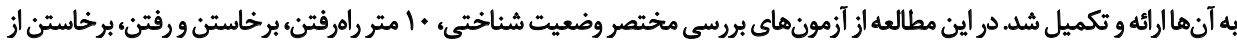

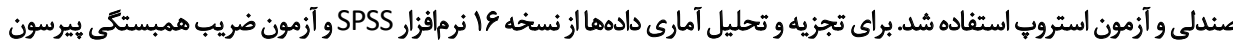

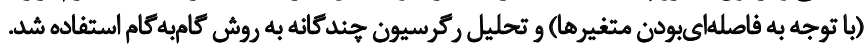

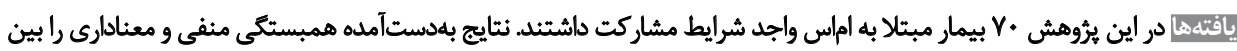

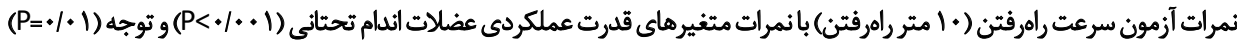

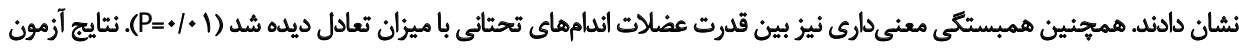

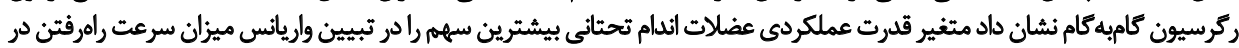
اين افراد داشته است.

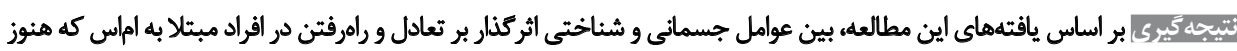

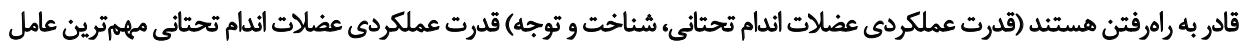

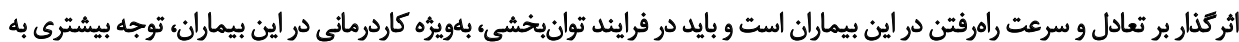
برئامه تقويت عضلات اندامهاى تحائ تحتانى شود.
\end{abstract}

تاريخ دريافت: 19 شهريور IT9V

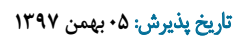

تاريخ انتشار: rا فروردين هونه

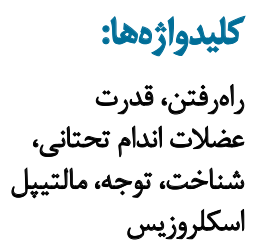

ㅁ. 
حسىييكرى، تنظيم و تبديل آن به دستورات حركتى براى

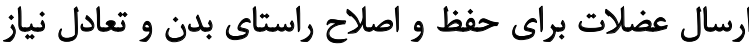

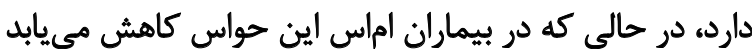

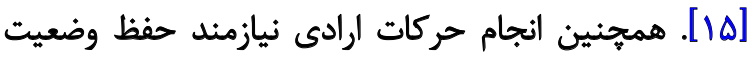

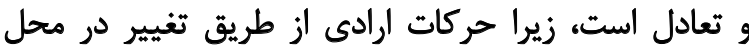

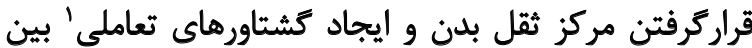

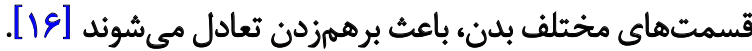

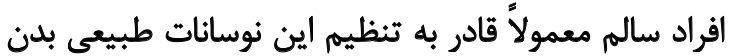

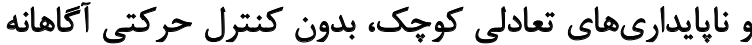

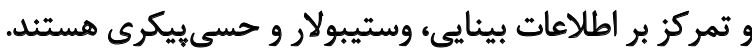

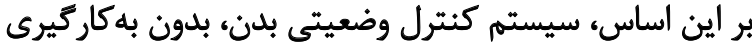

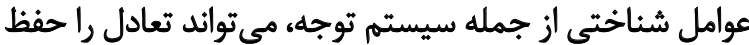

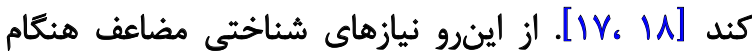

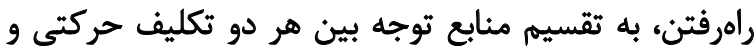

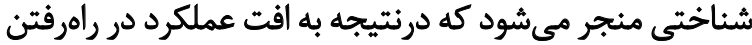

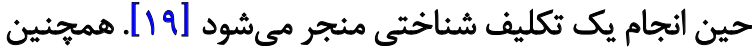

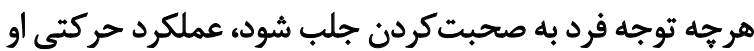

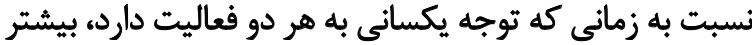

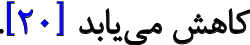
در افراد دجار اماس توانايى حفظ تعادل با متغيرهاى إنى إني

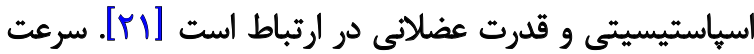

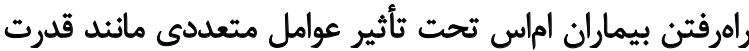

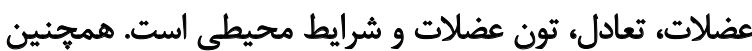

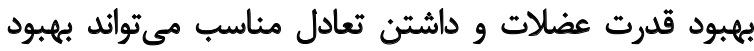

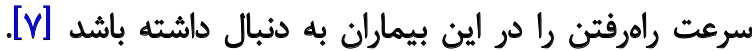

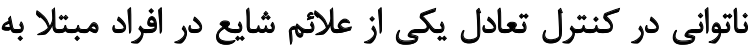

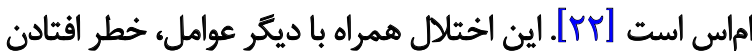

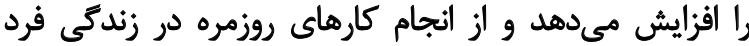

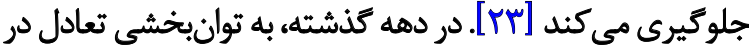

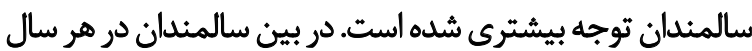

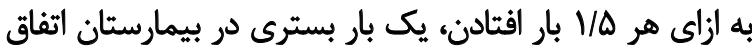

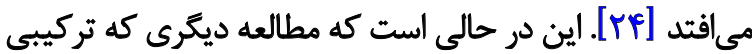

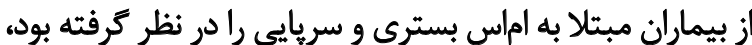

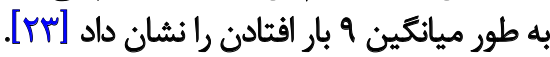

ارزيابى جنبههاي مختلف نقايص حركتى و تعيين دقيق

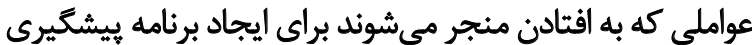

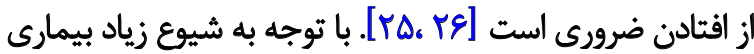

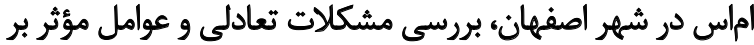

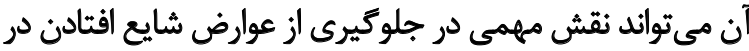

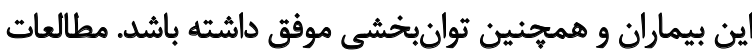
ذكرشده، عوامل مؤثر بر عملكرد راهرفتن و حفظ مون تعادل رادئ بادر افراد

\section{Interaction torques}

مقدمه

بيمارى مالتييل اسكلروزيس يا اماس بيمارى مزمن دستكاه

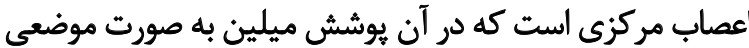

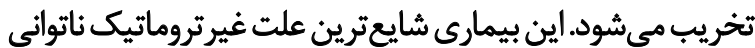

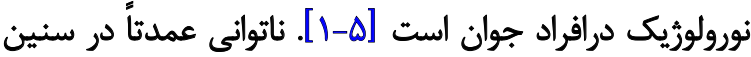

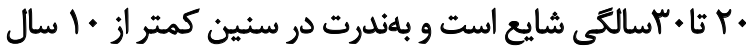

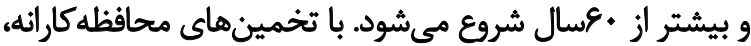

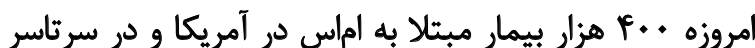

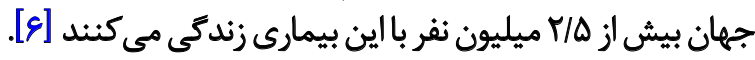
از آنجا كه اماس نواحى مختلف دستًاه عصبى را دركير

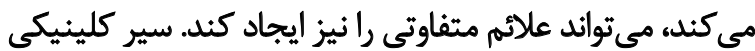

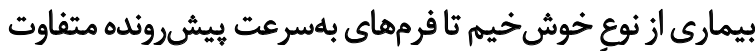

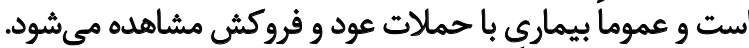

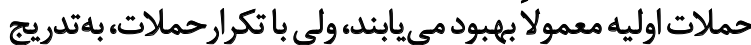

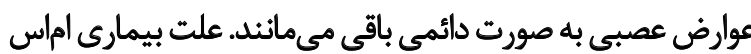
ناشناخته است. در مطالعات انجامشده سازوكارئ دارهاى اتوايميون،

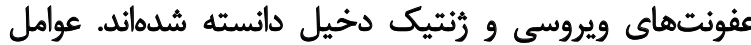

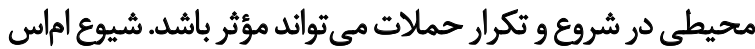

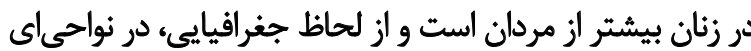
كه از خط استوا دورتر هستند بيشتر ديده مي جيود. از آنجا كه بيماران اماس باطيف كستردماى از علائم كرفتارى

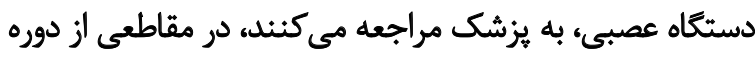

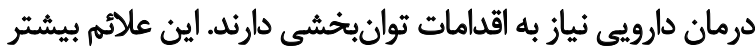

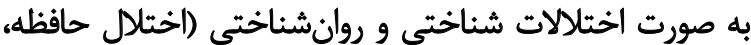

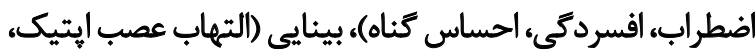

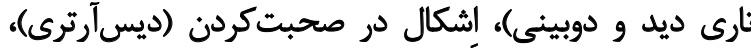

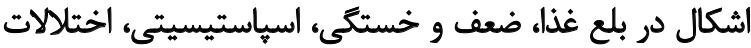

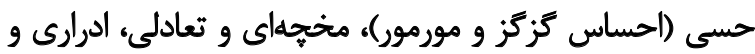

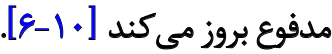

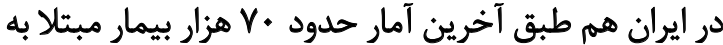

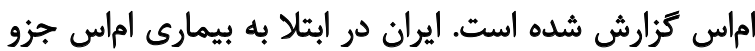

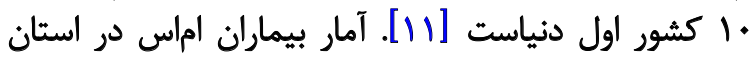

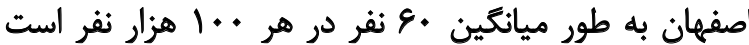
كه از ميانكين جهانى نيز بيشتر است. بر برانين اساس مطالعات

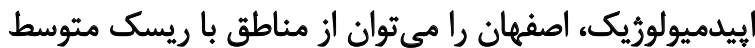

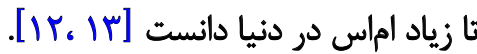
اختّلال تعادلى از ناتوانكنيندةترين علائم اين بيمارى است

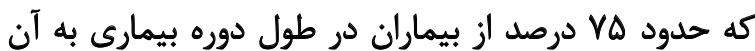

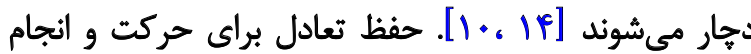

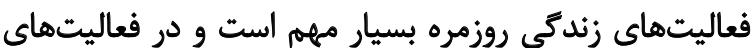

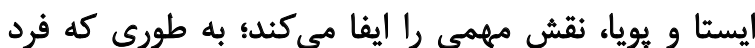

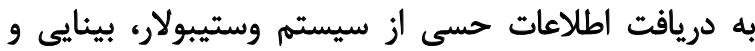


شخصى دريافتى از آنها محرمانه باقى خواهد ماند. مشاركت

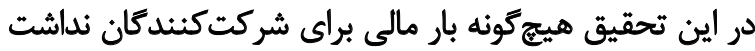

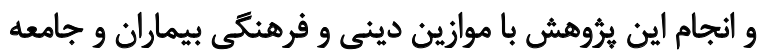

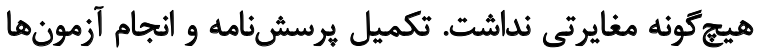

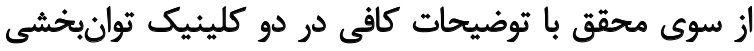

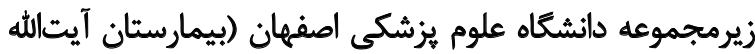

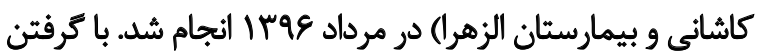

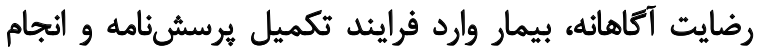

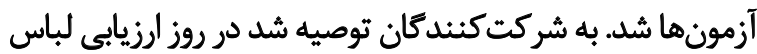

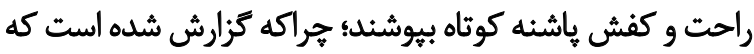

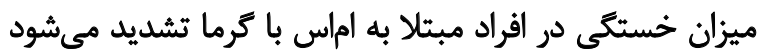
[r人]

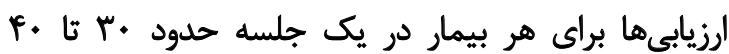

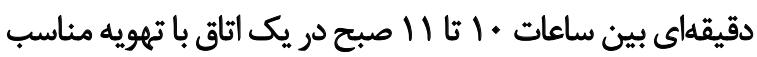

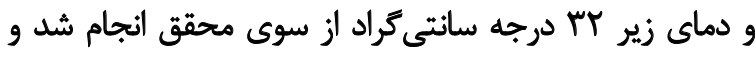

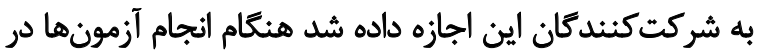

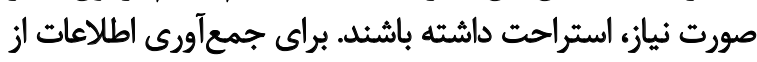

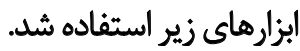

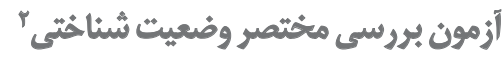

اين ابزار را ابتدا در سال 19vه فولشتين" به عنوان ضميمه

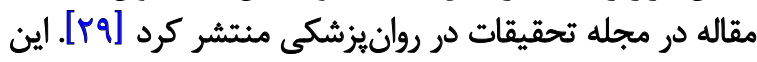

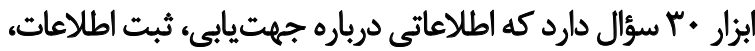

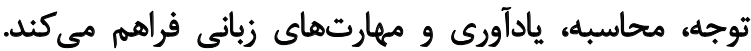

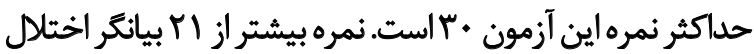

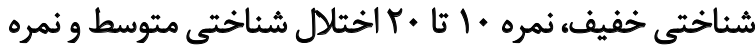

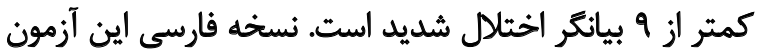

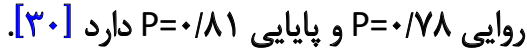

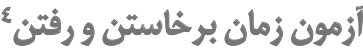

اين آزمون براى اندازهكيرى تعادل ديناميك و نشاندهنديه

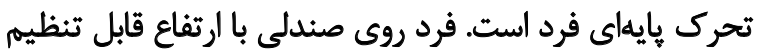

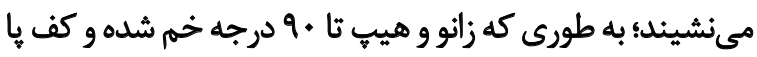

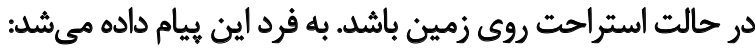

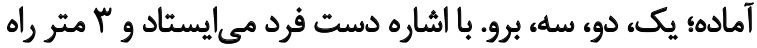

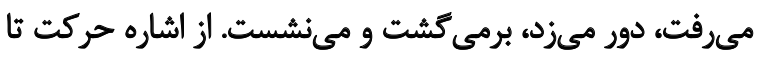

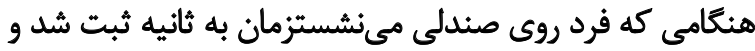

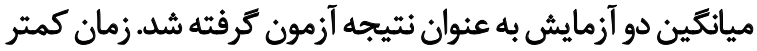

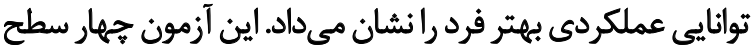

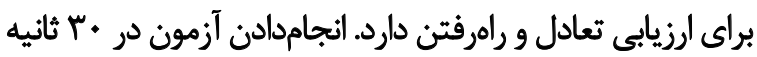

2. Mini-Mental State Examination (MMSE)

3. Folstein

4. Time Up \& Go test (TUG)
دجار اماس به طور مجزا بررسى كردهانده اما با توجه به اينكه

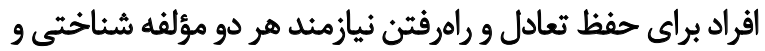

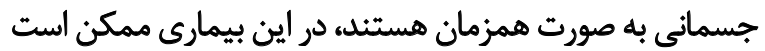

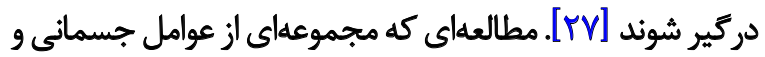

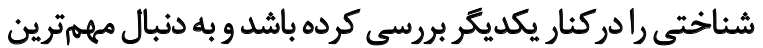

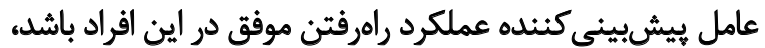

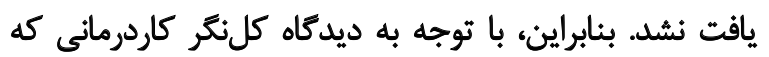
دريافت كنند

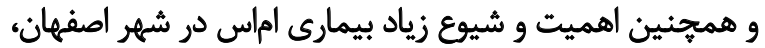

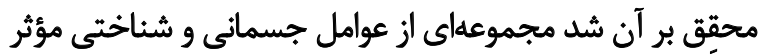
بر عملكرد راهرفتن (قدرت عضلات اندام تحتانى، شناخت و توجئه رادر اين بيماران بررسى كند.

$$
\text { ورش برورى }
$$

اين بُؤهش از نوع همبستخىتحليلى بود و در آن از روش

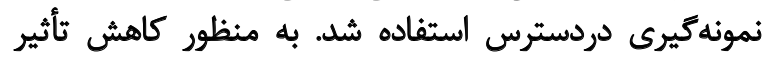

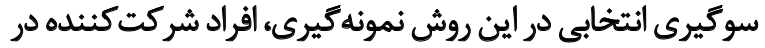

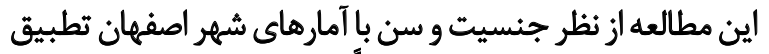

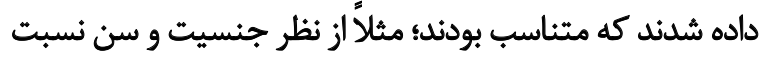

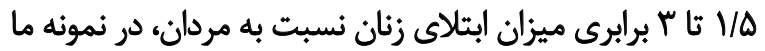

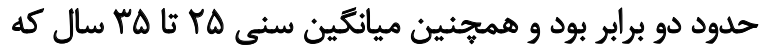

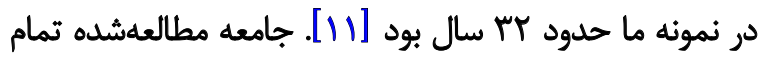

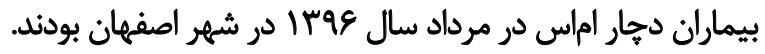

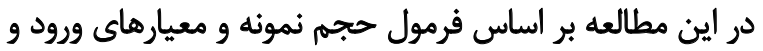

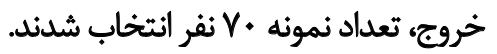

$70=N \geq\left[\frac{2-2 p^{2}+\varepsilon}{\varepsilon}\right](K+1)$

زين (1991) قاعده سرانغشتى را براى حداقل حجم نمون إنه در

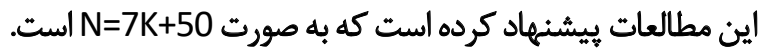

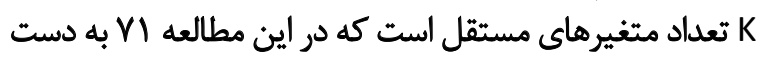

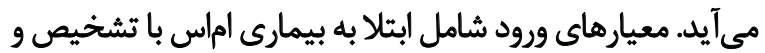

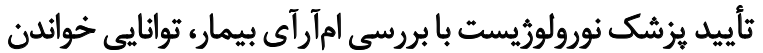

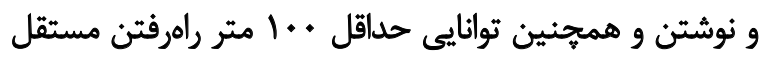

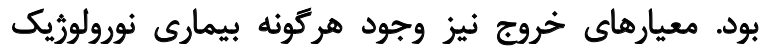

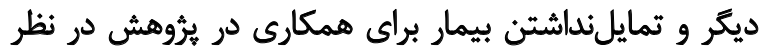

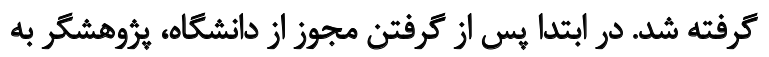

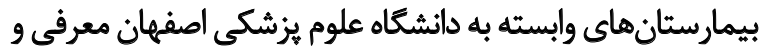
مجوز انجام تحقيق از مديريت مراكز كرفته شد.

يس از مطالعه يرونده يزشكى و بادرنظر برفتن معيار هاى ورود

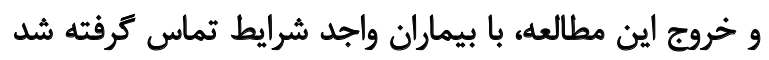

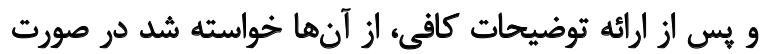

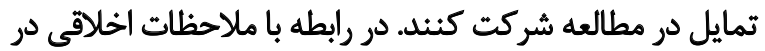

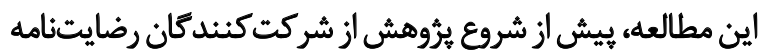

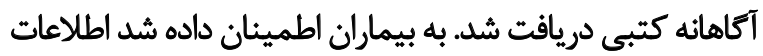


بوده، انجام مي داد. تعداد تكرارهاي انجامشده به عنوان امتياز

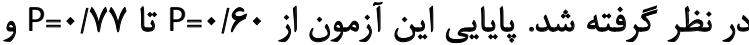

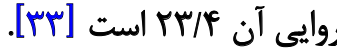

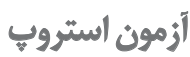

اثر استروبّا، به نام جان ريدلى استروب نامَّذارى شده است

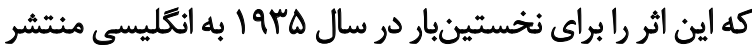

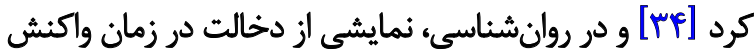

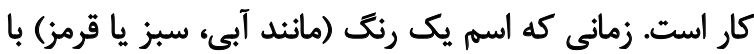

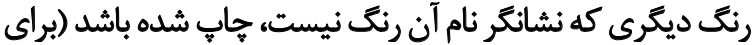

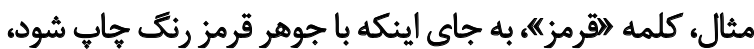

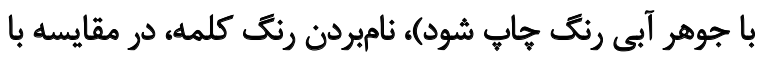

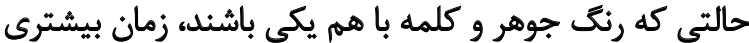

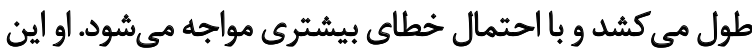

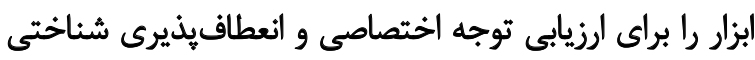

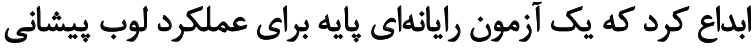

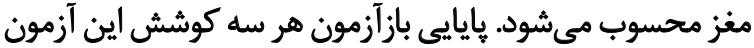

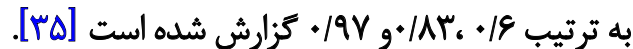

براى تجزيه و تحليل آمارى دادهها از نسخه 9 ا نرمافزٔار آمارى

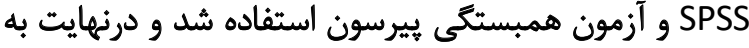

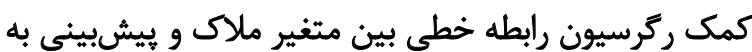

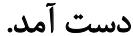

يافتهها

در اين مطالعه · V. بيمار مبتلا به اماس شركت داشتند. از

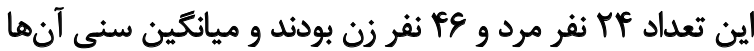

\section{Stroop effect}

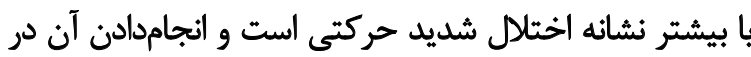

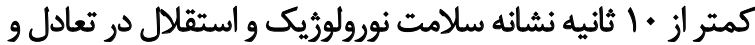

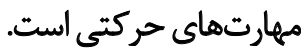

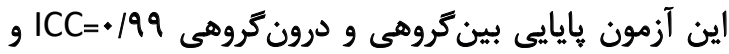

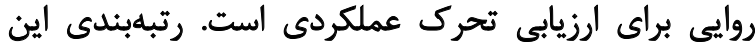

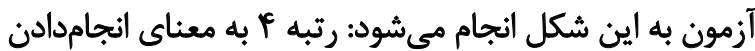

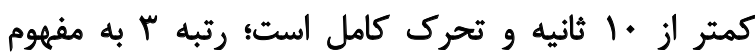

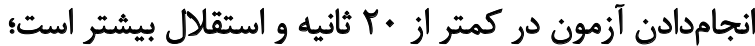

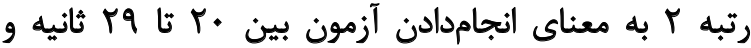

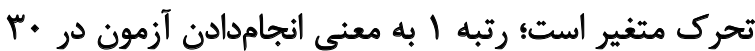

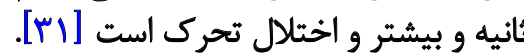

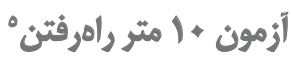

اين آزمون براى سنجش سرعت راهرفتن در اين يُروهش به بردي

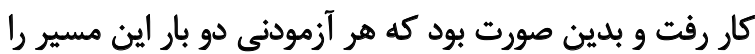

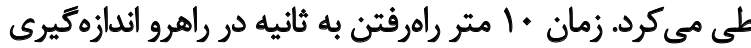

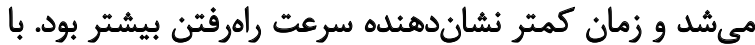

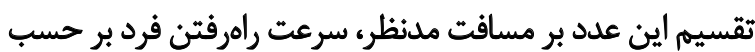

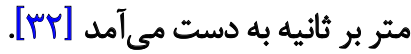

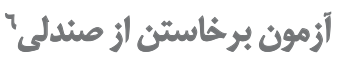

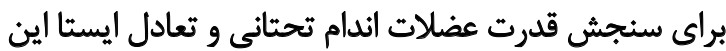

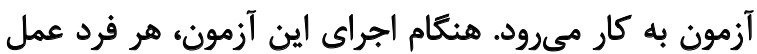

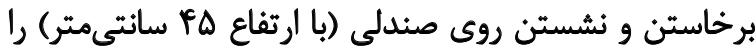

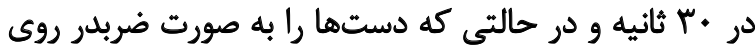

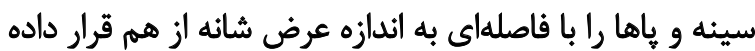

5. Ten Meter Walk Test

6. Sit to Stand Test

جدول ا. ويرّكى هاى جمعيتشناختى افراد شركت كنيده در مطالعه

\begin{tabular}{|c|c|c|c|c|c|}
\hline انحراف معيار & مياتكين & ل حرصد & فراوانى & شاخص آمارى & متغير \\
\hline $9 / V$ & rollta & - & - & \multirow{2}{*}{ - } & \multirow[b]{2}{*}{ سن } \\
\hline VNA & MTRAT & - & - & & \\
\hline - & - & $\pi \in / r$ & $m$ & مرد & \multirow{2}{*}{ جنس } \\
\hline - & - & $g \Delta / V$ & pe & زن & \\
\hline - & - & $r V /$ & 19 & سيكل وكمتر & \multirow{4}{*}{ تحصيلات } \\
\hline - & - & rNG & rV & دييلم و فوق دييلم & \\
\hline - & - & $\pi / q$ & $M$ & لسلائس & \\
\hline - & - & $1 / f$ & 1 & فوق ليسائس و بيشتر & \\
\hline
\end{tabular}


جدول r. همبستكى بين قدرت، شناخت و توجه بر عملكرد راهرفتن در افراد مبتلا به اماس

\begin{tabular}{|c|c|c|c|}
\hline سطح معنى دارى & ضريب همبستكى & شاخص آمارى & متغير \\
\hline$\% 18$ & $-\bullet / T M$ & قلدرث & \\
\hline . $/ M r$ & $-+1+11$ & شناخت & تعادل \\
\hline $1.0 \mathrm{~V}$ & $=\pi$ & توجه & \\
\hline .1 .01 & $-. / P \Delta)^{\circ}$ & قلرت & \\
\hline . 1199 & -.1 .10 & شناخت & سرعت \\
\hline .1011 & $=/ r+r$ & توجه & \\
\hline
\end{tabular}

توانبخننى

جدول بّ. نتايج تحليل ركرسيون كامبه كام مؤلفههاي بيشبينى كننده با سرعت راهرفتن در افراد مبتلا به اماس

\begin{tabular}{|c|c|c|c|c|c|}
\hline معناذارى & ضريب F F & خطاى استائدارد & ضريب R تعديلشده & ضريب R & مثغير هاى ييشيين \\
\hline .10 .1 & IV/giq & $\Delta / \Delta F$ &.$/ 194$ & $+\pi+9$ & قدرث أندام تحتثاني \\
\hline
\end{tabular}

جدول F. ضرايب ركرسيون براى مؤلفه بيشبينى كثنده ميزان سرعت راهرفتن در افراد مبتلا به اماس

\begin{tabular}{|c|c|c|c|c|c|}
\hline $\mathbf{P}$ & $\mathbf{T}$ & S.E & Beta & B & شاخصآمارى \\
\hline $.1 . .1$ & NTMA & & $\pi / \cdot \pi$ & $|V| \cdot 1 \Delta$ & مقدار ثابت \\
\hline $.1 . .1$ & $4 / 14$ & - RAAF &.$/ N K A$ & . /orq & قدرت اندام تحتثانى \\
\hline
\end{tabular}

تحتاني وارد شده است و نمرات توجه به دليل اينكه رابطه كم

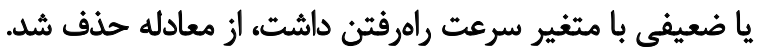

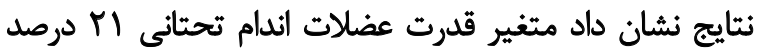

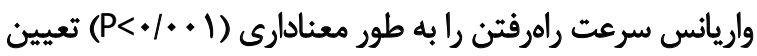

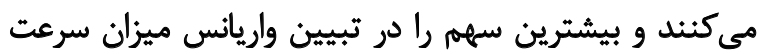

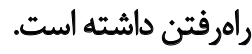

ثک

اين مطالعه با هدف تعيين مؤلفههاى اثرگذار بر راهرفتن

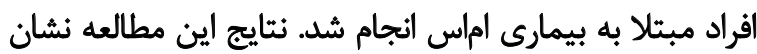

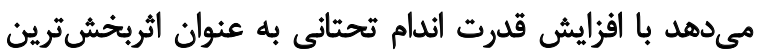

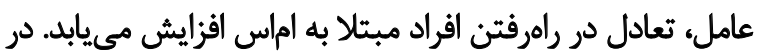

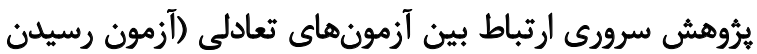

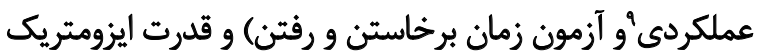

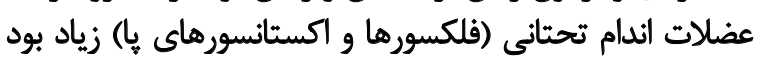

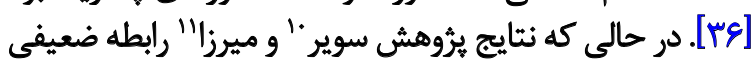

9. Functional Reach Test (FRT)

10. Soyuer

11. Mirza
مطالعه بلد/DD

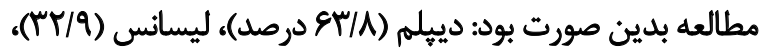

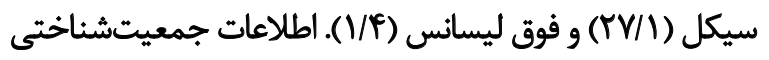

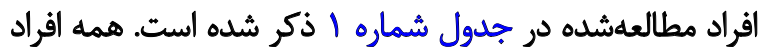

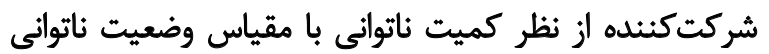

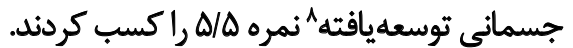

به منظور بررسى رابطه قدرت عضلات اندامهاى تحتانى، وضعيت شناختي، توجه و سرعت با تعادل افراد مبتلا بيه اماس،

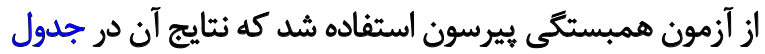

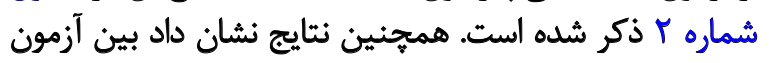

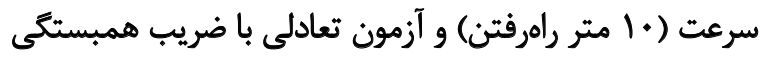

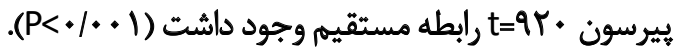

به منظور هيشبيني و تبيين ميزان سرعت راهرفتن افراد

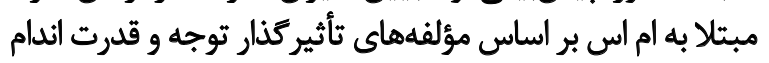
تحتانى از تحليل ركرسيون جيند

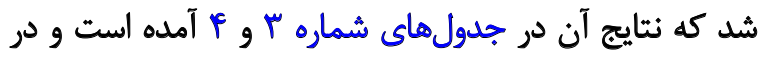

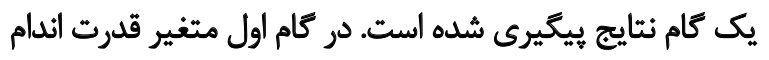

8. Expanded Disability Status Scale (EDSS) 
بيشتر از •وسال مبتلابه سكته مغزى رابررسى كرد. نتايج حاصل

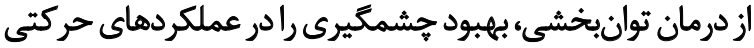

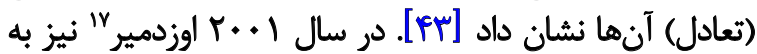

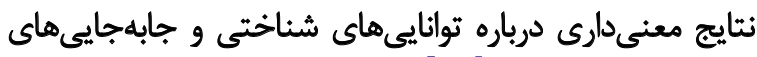

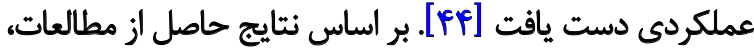

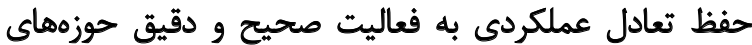

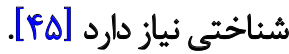

براي بهبود تعادل، ارزيابى و درمان حوزههاى شناختى اهميت

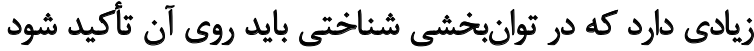

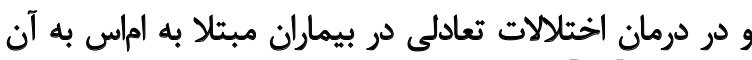

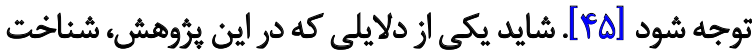

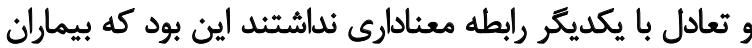

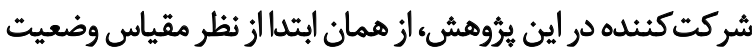

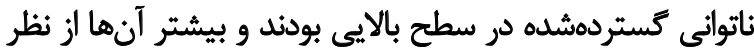

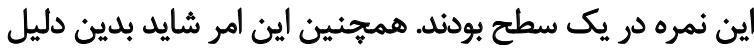

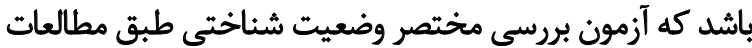

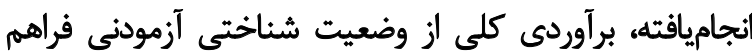

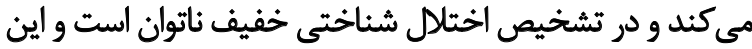

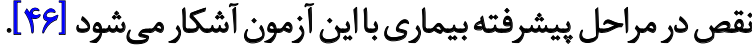

همجنين نشان داده شده است نارسايىهاى توجهي موجب

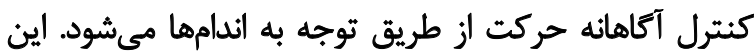

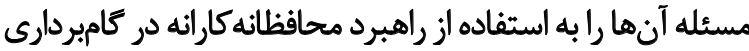

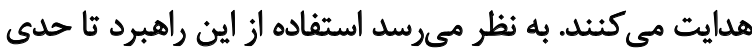

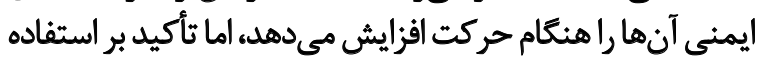

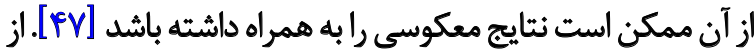

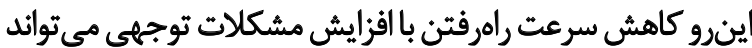

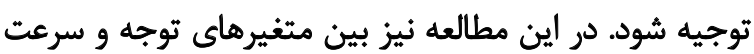

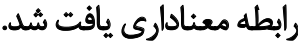

\section{نتيجليَيرى}

بر اساس نتايج مطالعه، ازديدگًاه كلنكَر كاردرمانى از بين عوامل

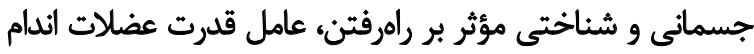

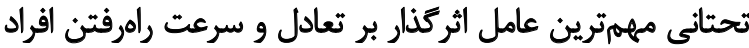

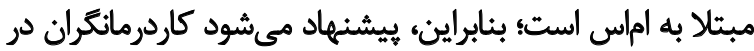

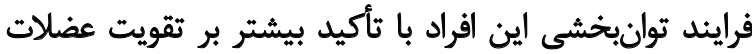

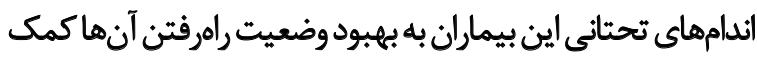

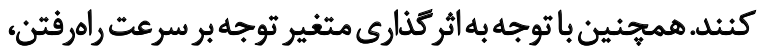

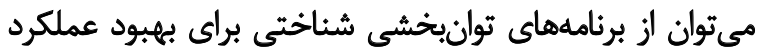

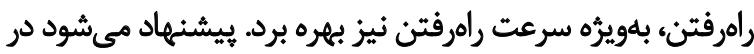

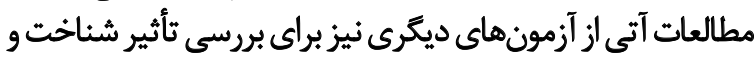
تعادل بر عملكرد راهرفتن اين بيماران استفاده شود.

\section{7. Özdemir}

را بين آزمونهاى تعادلى و قدرت عضلات اندام تحتاني نشان

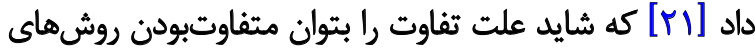

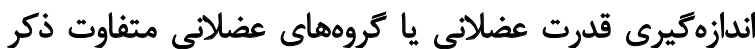

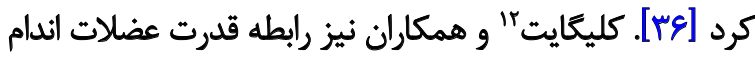

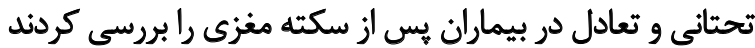

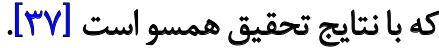

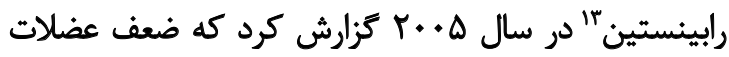

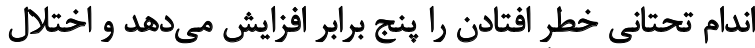

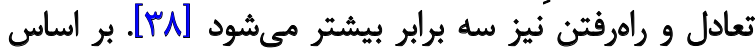

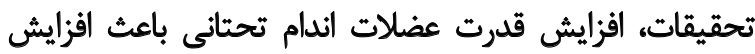

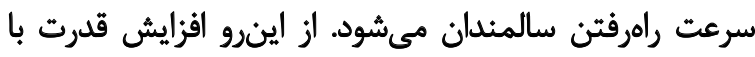

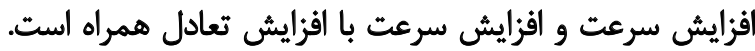

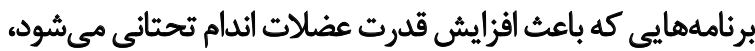

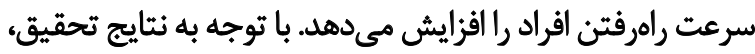

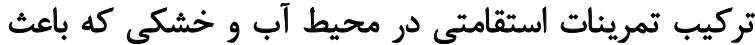

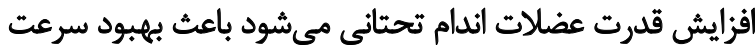

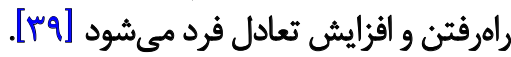
تغييريذيرى سرعت به عنوان شاخص تغييريذيرى كلى،

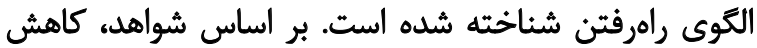

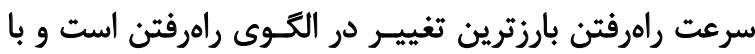

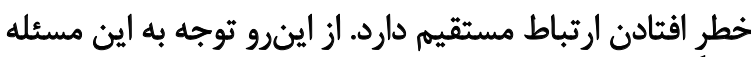

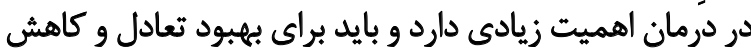

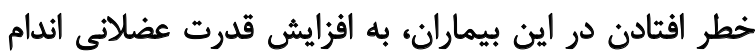

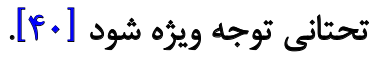
همرين" و وهماران و بوهانون "1ه رابطه ضعيفى بين قدرت

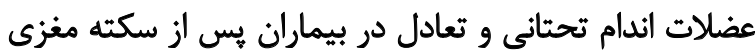

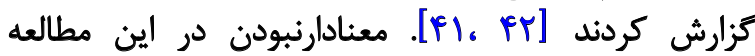

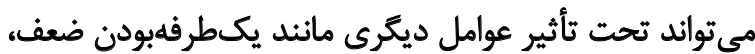

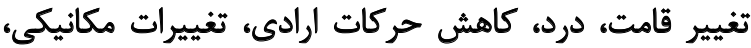

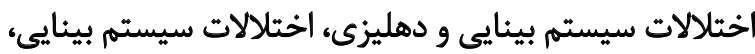

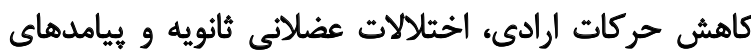

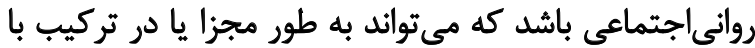

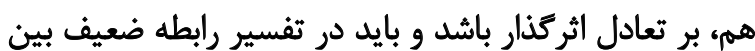

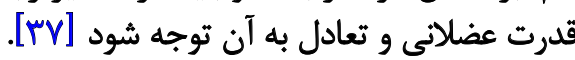

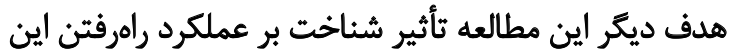

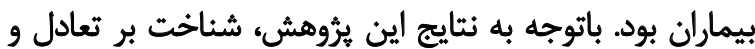

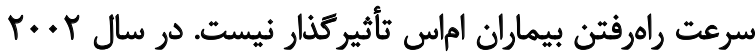

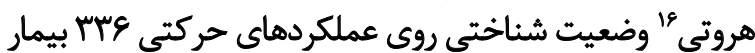

\section{Kligyte}

13. Rubenstein

14. Hamrin

15. Bohanon

16. Heruti 
از محدوديتهاي اين مطالعه مي توان به اين نكثه اشاره كرد كه رونه

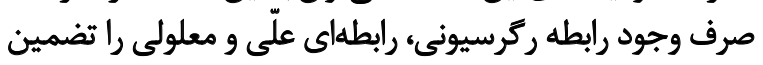

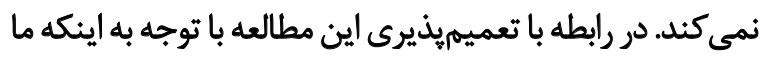

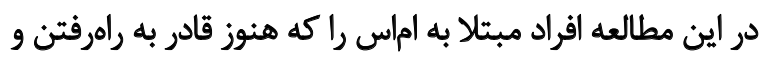

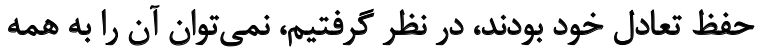
بيماران اماس، بهويثه افراد ناتوانتر تعميم داد.

ماحظات أخلاقى

يبيروى از اصول الخاق يُوهش

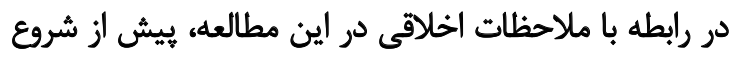

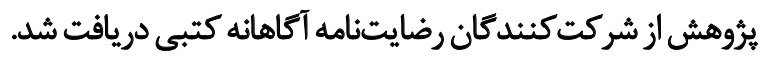

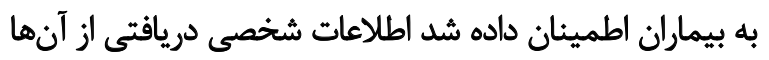

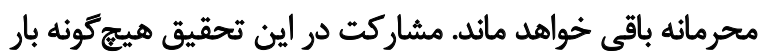

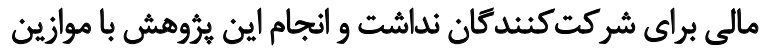

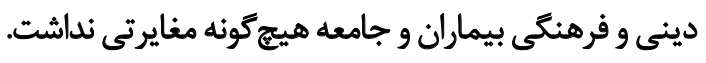

$$
\text { مامى مالى }
$$

اين مقاله حامى مالى ندارد.

مشاركت نويسئد مَان

مشاركت نويسندكان به شرح زير است: نهايیىسازى: سيد على مئى

حسينى؛ مجرى طرح: امين مهدىز اديه.

تعارض مناقع

بنابر اظهار نويسندكان، اين مقاله تعارض منافع ندارد. 


\section{References}

[1] Ellwardt E, Zipp F. Molecular mechanisms linking neuroinflammation and neurodegeneration in MS. Experimental Neurology. 2014; 262(A):8-17. [DOI:10.1016/j.expneurol.2014.02.006] [PMID]

[2] Friese MA, Schattling B, Fugger L. Mechanisms of neurodegeneration and axonal dysfunction in Multiple Sclerosis. Nature Reviews Neurology. 2014; 10(4):225-38. [DOI:10.1038/nrneurol.2014.37] [PMID]

[3] Kutzelnigg A, Lassmann H. Pathology of Multiple Sclerosis and related inflammatory demyelinating diseases. Handbook of Clinical Neurology. 2014; 122:15-58. [DOI:10.1016/B978-0444-52001-2.00002-9] [PMID]

[4] Lassmann H, Van Horssen J, Mahad D. Progressive Multiple Sclerosis: Pathology and pathogenesis. Nature Reviews Neurology. 2012; 8(11):647-56. [DOI:10.1038/nrneurol.2012.168] [PMID]

[5] Trapp BD, Nave KA. Multiple Sclerosis: An immune or neurodegenerative disorder. Annual Review of Neuroscience. 2008; 31:247-69. [DOI:10.1146/annurev.neuro.30.051606.094313] [PMID]

[6] Cameron MH, Wagner JM. Gait abnormalities in Multiple Sclerosis: Pathogenesis, evaluation, and advances in treatment. Current Neurology and Neuroscience Reports. 2011; 11(5):507-15. [DOI:10.1007/s11910-011-0214-y] [PMID]

[7] Fjeldstad C, Pardo G, Frederiksen C, Bemben D, Bemben M. Assessment of postural balance in Multiple Sclerosis. International Journal of MS Care. 2009; 11(1):1-5. [DOI:10.7224/15372073-11.1.1]

[8] Kamalian Lari S, Haghgoo HA, Farzad M, Hosseinzadeh S. [Investigation of the validity and reliability of Balance Evaluation Systems Test (BESTest) in assessment of balance disorders in people with Multiple Sclerosis (Persian)]. Archives of Rehabilitation. 2018; 18(4):288-95. [DOI:10.21859/jrehab.18.4.3]

[9] A'zimian M. [Review: Osychological \& physical rehabilitation in Multiple Sclerosis (Persian)]. Archives of Rehabilitation. 2001; 1(3):43-7.

[10] Mehraban S, Bahmani B, Azimian M, Rezasoltani P. The Effectiveness of Cognitive-Behavioral-Based Stress Management Training on Anxiety in Female MS Patients. Iranian Rehabilitation Journal. 2015; 13(3):49-53.

[11] Etemadifar M, Sajjadi S, Nasr Z, Firoozeei TS, Abtahi SH, Akbari $\mathrm{M}$, et al. Epidemiology of Multiple Sclerosis in Iran: A systematic review. European Neurology. 2013; 70(5-6):356-63. [DOI:10.1159/000355140] [PMID]

[12] Etemadifar M, Janghorbani M, Shaygannejad V, Ashtari F. Prevalence of Multiple Sclerosis in Isfahan, Iran. Neuroepidemiology. 2006; 27(1):39-44. [DOI:10.1159/000094235] [PMID]

[13] Etemadifar $M$, Maghzi A-H. Sharp increase in the incidence and prevalence of Multiple Sclerosis in Isfahan, Iran. Multiple Sclerosis Journal. 2011; 17(8):1022-7. [DOI:10.1177/1352458511401460] [PMID]
[14] Findling O, Sellner J, Meier N, Allum JH, Vibert D, Lienert C, et al. Trunk sway in mildly disabled Multiple Sclerosis patients with and without balance impairment. Experimental Brain Research. 2011; 213(4):363. [DOI:10.1007/s00221-011-2795-8] [PMID]

[15] Doumas M, Smolders C, Krampe RT. Task prioritization in aging: Effects of sensory information on concurrent posture and memory performance. Experimental Brain Research. 2008; 187:275. [DOI:10.1007/s00221-008-1302-3] [PMID]

[16] Ting LH. Dimensional reduction in sensorimotor systems: A framework for understanding muscle coordination of posture. Progress in Brain Research. 2007; 165:299-321. [DOI:10.1016/ S0079-6123(06)65019-X]

[17] Riley MA, Baker AA, Schmit JM. Inverse relation between postural variability and difficulty of a concurrent short-term memory task. Brain Research Bulletin. 2003; 62(3):191-5. [DOI:10.1016/j.brainresbull.2003.09.012] [PMID]

[18] Shumway-Cook A, Woollacott MH. Motor control: Translating research into clinical practice. Philadelphia: Lippincott Williams \& Wilkins; 2007. [PMCID]

[19] Verghese J, Buschke H, Viola L, Katz M, Hall C, Kuslansky G, et al. Validity of divided attention tasks in predicting falls in older individuals: A preliminary study. Journal of the American Geriatrics Society. 2002; 50(9):1572-6. [DOI:10.1046/j.15325415.2002.50415.x] [PMID]

[20] Verghese J, Kuslansky G, Holtzer R, Katz M, Xue X, Buschke $\mathrm{H}$, et al. Walking while talking: Effect of task prioritization in the elderly. Archives of Physical Medicine and Rehabilitation. 2007; 88(1):50-3. [DOI:10.1016/j.apmr.2006.10.007] [PMID] [PMCID]

[21] Soyuer F, Mirza M. [Relationship between lower extremity muscle strength and balance in Multiple Sclerosis (Turkish)]. Journal of Neurological Sciences. 2006; 23(4):257-63.

[22] Corradini ML, Fioretti S, Leo T, Piperno R. Early recognition of postural disorders in Multiple Sclerosis through movement analysis: a modeling study. IEEE Transactions on Biomedical Engineering. 1997; 44(11):1029-38. [DOI:10.1109/10.641330] [PMID]

[23] Cattaneo D, De Nuzzo C, Fascia T, Macalli M, Pisoni I, Cardini R. Risks of falls in subjects with Multiple Sclerosis. Archives of Physical Medicine and Rehabilitation. 2002; 83(6):864-7. [DOI:10.1053/apmr.2002.32825] [PMID]

[24] Society AG, Society G, Of AA, On Falls Prevention OSP. Guideline for the prevention of falls in older persons. Journal of the American Geriatrics Society. 2001; 49(5):664-72. [DOI:10.1046/j.1532-5415.2001.49115.x]

[25] DeBolt LS, McCubbin JA. The effects of home-based resistance exercise on balance, power, and mobility in adults with Multiple Sclerosis. Archives of Physical Medicine and Rehabilitation. 2004; 85(2):290-7. [DOI:10.1016/j.apmr.2003.06.003]

[26] Cattaneo D, Cardini R. Computerized system to improve voluntary control of balance in neurological patients. CyberPsychology \& Behavior. 2001; 4(6):687-94. [DOI:10.1089/109493 101753376632] [PMID] 
[27] Hamilton F, Rochester L, Paul L, Rafferty D, O'leary C, Evans J. Walking and talking: An investigation of cognitive-motor dual tasking in Multiple Sclerosis. Multiple Sclerosis Journal. 2009; 15(10):1215-27. [DOI:10.1177/1352458509106712] [PMID]

[28] Krupp LB, Alvarez LA, LaRocca NG, Scheinberg LC. Fatigue in Multiple Sclerosis. Archives of Neurology. 1988; 45(4):435-7. [DOI:10.1001/archneur.1988.00520280085020] [PMID]

[29] Folstein MF, Folstein SE, McHugh PR. "Mini-Mental State": A practical method for grading the cognitive state of patients for the clinician. Journal of Psychiatric Research. 1975; 12(3):18998. [PMID]

[30] Seyedian M, Falah M, Nourouzian M, Nejat S, Delavar A, Ghasemzadeh $\mathrm{H}$. [Validity of the Farsi version of mini-mental state examination (Persian)]. Journal of Medical Council of Iran. 2008; 25(4):408-14.

[31] Schoppen T, Boonstra A, Groothoff JW, de Vries J, Göeken LN, Eisma WH. The Timed Up and Go Test: Reliability and validity in persons with unilateral lower limb amputation. Archives of Physical Medicine and Rehabilitation. 1999; 80(7):825-8. [DOI:10.1016/S0003-9993(99)90234-4]

[32] Mossberg KA. Reliability of a timed walk test in persons with acquired brain injury. American Journal of Physical Medicine \& Rehabilitation. 2003; 82(5):385-90. [DOI:10.1097/01. PHM.0000052589.96202.BE]

[33] Møller AB, Bibby BM, Skjerbæk AG, Jensen E, Sørensen H, Stenager $E$, et al. Validity and variability of the 5-repetition sitto-stand test in patients with Multiple Sclerosis. Disability and Rehabilitation. 2012; 34(26):2251-8. [DOI:10.3109/09638288. 2012.683479] [PMID]

[34] Stroop JR. Studies of interference in serial verbal reactions. Journal of Experimental Psychology. 1935; 18(6):643-62. [DOI:10.1037/h0054651]

[35] Ghadiri F, Jazayeri A, Ashaeri H, Ghazi Tabatabaei M. Deficit in executive functioning in patients with schizo-obsessive disorder. Advances in Cognitive Science. 2006; 8(3):11-24.

[36] Ebrahimi Atri A, Sarvari F, Saeedi M, Khorshid Sokhangu M. [Relationship between lower extremity muscle strength and dynamic balance in women with Multiple Sclerosis (MS) (Persian)]. Journal of Research in Rehabilitation Sciences. 2013; 9(1):20-7.

[37] Kligyte I, Lundy-Ekman L, Medeiros JM. [Relationship between lower extremity muscle strength and dynamic balance in people post-stroke (Lithuanian)]. Medicina (Kaunas). 2003; 39(2):122-8. [PMID]

[38] Rubenstein LZ, Josephson KR, Trueblood PR, Loy S, Harker JO, Pietruszka FM, et al. Effects of a group exercise program on strength, mobility, and falls among fall-prone elderly men. The Journals of Gerontology Series A: Biological Sciences and Medical Sciences. 2000; 55(6):M317-M21. [DOI:10.1093/ gerona/55.6.M317] [PMID]

[39] Sadeghi H, Ghasempoor H, Yadegaripoor M, Shojaedin S. [The effect of eight week combined aquatic and non-aquatic training program on the lower extremity strength and gait speed of elderly men (Persian)]. Iranian Journal of Ageing. 2013; 7(4):59-66.
[40] Brach JS, Berthold R, Craik R, VanSwearingen JM, Newman $A B$. Gait variability in community-dwelling older adults. Journal of the American Geriatrics Society. 2001; 49(12):1646-50. [DOI:10.1111/j.1532-5415.2001.49274.x] [PMID]

[41] Bohannon RW. Standing balance, lower extremity muscle strength, and walking performance of patients referred for physical therapy. Perceptual and Motor Skills. 1995; 80(2):37985. [DOI:10.2466/pms.1995.80.2.379] [PMID]

[42] Hamrin E, Eklund G, Hillgren AK, Borges O, Hall J, Hellström $O$. Muscle strength and balance in post-stroke patients. Upsala Journal of Medical Sciences. 1982; 87(1):11-26. [DOI:10.3109/03009738209178405] [PMID]

[43] Heruti RJ, Lusky A, Dankner R, Ring H, Dolgopiat M, Barell V, et al. Rehabilitation outcome of elderly patients after a first stroke: Effect of cognitive status at admission on the functional outcome. Archives of Physical Medicine and Rehabilitation. 2002; 83(6):742-9. [DOI:10.1053/apmr.2002.32739] [PMID]

[44] Özdemir F BM, Tabatabaei R, Ekuklu G, Kokino S. Cognitive evaluation and functional outcome after stroke. American Journal of Physical Medicine \& Rehabilitation. 2001; 80(6):4105. [DOI:10.1097/00002060-200106000-00003]

[45] Soltanlou M, Anbara T, Taghi-Zadeh G, Rahim-Zadeh Rahbar $\mathrm{S}$, Karimi $\mathrm{H}$. [Assessing the relationship between cognitive deficits and functional balance in right adult stroke patients (Persian)]. Urmia Medical Journal. 2013; 24(5):295-301.

[46] Chehrehnegar N, Shams F, Zarshenas S, Kazemi F. [Evaluating the reliability of the Montreal cognitive assessment test and its agreement with mini mental state examination among healthy elderly (Persian)]. Journal of Research in Rehabilitation Sciences. 2011; 7(5):674-80.

[47] Wulf G, McNevin N, Shea CH. The automaticity of complex motor skill learning as a function of attentional focus. The Quarterly Journal of Experimental Psychology: Section A. 2001; 54(4):1143-54. [DOI:10.1080/713756012] [PMID] 